\title{
Jahresinhaltsverzeichnis 54. Jahrgang 2006
}

\section{ARTIKEL}

Saskia Böcking

Patrick Donges

Martin Emmer / Angelika Füting / Gerhard Vowe

Jörg Hagenah

Jörg Hagenah

Wolfgang Hoffmann-Riem

Kurt Imhof

Nikolaus Jackob
Elterlicher Umgang mit kindlicher Fernsehnutzung. Test einer deutschsprachigen Skala und erste Befunde für die Deutschschweiz . . . . . . . . . . . . . . 4/599

Medien als Institutionen und ihre Auswirkungen auf Organisationen. Perspektiven des soziologischen Neo-Institutionalismus für die Kommunikationswissenschaft ....................... 4/563

Wer kommuniziert wie über politische Themen? Eine empirisch basierte Typologie individueller politischer Kommunikation

Möglichkeiten der Nutzung von Media-AnalyseFernsehdaten für Sekundäranalysen von 1972 bis heute ....................... 4/637

Möglichkeiten der Nutzung von Media-AnalyseRadiodaten für Sekundäranalysen von 1972 bis heu te ............................ 3/457

Rundfunk als Public Service. Anmerkungen zur Vergangenheit, Gegenwart und Zukunft öffentlich-rechtlichen Rundfunks .................. 1/95

Mediengesellschaft und Medialisierung $2 / 191$

Macht und Verantwortung der Kommunikation bei Cicero. Ein historischer Beitrag zum Ethikdiskurs in der Kommunikationswissenschaft

Kathrin Junghanns / Thomas Hanitzsch Deutsche Auslandskorrespondenten im Profil . . 3/412

Veronika Karnowski / Thilo von Pape / Zur Diffusion Neuer Medien: Kritische BestandsWerner Wirth aufnahme aktueller Ansätze und Überlegungen zu einer integrativen Diffusions- und Aneignungstheorie Neuer Medien .................. 1/56

Christoph Klimmt / Kerrin Bartels / Helmut Scherer

Kommunikation für die Rundfunkgebühr. Die Furchtappelle der Gebühreneinzugszentrale fruchten weniger als Überzeugungsarbeit ......... 4/579

Fragmentierte Publika in der Transformationsgesellschaft Estlands. Tendenzen der Mediennutzung . . 4/620

Ragne Kõuts-Klemm 
Henk Erik Meier

Jutta Milde / Georg Ruhrmann

Oliver Quiring / Wolfgang Schweiger

Ulrich Riehm / Bettina-Johanna Krings

Holger Schramm / Werner Wirth

Wolfgang Seufert

Jens Wolling / Christoph Kuhlmann
Die Regulierungskrise des öffentlich-rechtlichen Rundfunks ....................... 2/258

Molekulare Medizin in deutschen TV-Wissenschaftsmagazinen. Ergebnisse von Journalisteninterviews und Inhaltsanalysen $\ldots \ldots \ldots \ldots \ldots . .3 / 430$

Interaktivität - ten years after. Eine Bestandsaufnahme und ein Analyserahmen ...............

Abschied vom „Internet für alle“? Der „blinde Fleck“ in der Diskussion zur digitalen Spaltung ..

MedienundEmotionen. Bestandsaufnahmeeinesvernachlässigten Forschungsfeldes aus medienpsychologischer Perspektive ...................

Programmaufwand, Qualität und Wirtschaftlichkeit öffentlich-rechtlicher Rundfunkangebote . . . 3/365

Zerstreute Aufmerksamkeit. Empirischer Test eines Erklärungsmodells für die Nebenbeinutzung des Fernsehens ...................... 3/386

\section{LITERATUR}

Reihe „Klassiker der Kommunikations- und Medienwissenschaft heute“

Udo Göttlich

Maren Hartmann

\section{Besprechungen}

Joan Kristin Bleicher

Joan Kristin Bleicher
Leo Löwenthal: Soziale Theorie der Massenkultur und kritische Kommunikationsforschung. Löwenthals Medienanalysen und Massenkulturkritik im Kontext der amerikanischen Kommunikationsforschung der Nachkriegszeit ................ 1/105

Der Kulturkritiker als Flaneur. Walter Benjamin, die Passage und die neuen (Medien-) Technologien ............................ 2/288
Abschiede von der Wirklichkeit. Aktuelle Frontlinien der medien- bzw. kommunikationswissenschaftlichen Fernsehforschung seit 2005 - eine Sammelrezension ..................... 4/654

Guido Zurstiege: Zwischen Kritik und Faszination. Was wir beobachten, wenn wir die Werbung beobachten, wie sie die Gesellschaft beobachtet. Köln: Herbert von Halem, 2005 ................. 2/308 
Roger Blum

Michel Clement

Indira Dupuis

Susanne Eggert

Susanne Fengler

Johannes Fromme

Ursula Ganz-Blättler

Joachim R. Höflich

Dagmar Hoffmann

Jutta Kehrer

Nathalie Kharina-Welke

Marcus S. Kleiner
Heinz Bonfadelli, Martina Leonarz, Werner A. Meier (Hrsg.): Informationsgesellschaft Schweiz. Medien, Organisationen und Öffentlichkeit im Wandel. Zürich: Seismo, 2004 ..................... 2/311

Christian Scholz (Hrsg.): Handbuch Medienmanagement. Berlin: Springer, 2006 . . . . . . . . . . 4/667

Hans-Jörg Trenz: Europa in den Medien. Die europäische Integration im Spiegel nationaler Öffentlichkeit. Frankfurt: Campus, 2005 . . . . . . . . . . . 2/311

Emine Uçar-İlbuğa: Fernsehkonsum von türkischen Jugendlichen. Eine empirische Untersuchung im Hamburger Stadtteil Dulsberg. Frankfurt am Main: Lang, 2005

Gerd Hallenberger / Jörg-Uwe Nieland: Neue Kritik der Medienkritik. Werkanalyse, Nutzerservice, Sales Promotion oder Kulturkritik? Köln: Herbert von Halem, $2005 \ldots \ldots \ldots \ldots \ldots \ldots \ldots \ldots \ldots$. 1/128

Christine Feil/Regina Decker/Christoph Gieger: Wie entdecken Kinder das Internet? Beobachtungen bei 5- bis 12-jährigen Kindern. Wiesbaden: VS,

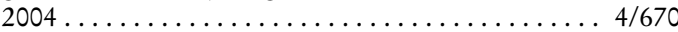

Tim Bergfelder: International Adventures. German Popular Cinema and European Co-Productions in the 1960s. New York / Oxford: Berghahn, 2005 .. 4/672

Michael Feldhaus: Mobile Kommunikation im Familiensystem. Zu den Chancen und Risiken mobiler Kommunikation für das familiale Zusammenleben. Würzburg: Ergon, 2004 ............... 2/313

Bertram Scheufele: Sexueller Missbrauch. Mediendarstellung und Medienwirkung. Wiesbaden: VS,

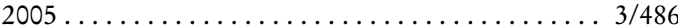

Thilo Büsching (Hrsg.): Mediengeschäftsmodelle der Zukunft. Baden-Baden: Nomos, 2005 ...... 4/674

Viktoria Brunmeier: Das Internet in Russland. Eine Untersuchung zum spannungsreichen Verhältnis von Politik und Runet. München: Fischer, 2005 .. 4/675

Rainer Winter (Hrsg.): Medienkultur, Kritik und Demokratie. Der Douglas Kellner-Reader. Köln: Herbert von Halem, 2005 . . . . . . . . . . . . . . . . . 2/315 
Hans J. Kleinsteuber

Daniela Kloock

Steffen Kolb / Steffen Burkhardt

Sven Kommer

Sonja Kretzschmar

Jan Krone

Hans-Dieter Kübler

Hans-Dieter Kübler

Thomas Lietz

Maria Löblich

Ursula Maier-Rabler

Marianne Merkt
Christina Holtz-Bacha: Medienpolitik für Europa. Wiesbaden: VS, $2006 \ldots \ldots \ldots \ldots \ldots \ldots \ldots$. . 4/677

Joachim Knape (Hrsg.): Medienrhetorik. Tübingen: Attempto Verlag, 2005 ................... 4/678

Norbert Baumgärtner: Risiko- und Krisenkommunikation. Rahmenbedingungen, Herausforderungen und Erfolgsfaktoren, dargestellt am Beispiel der chemischen Industrie. München: Dr. Hut, 2005 . . . . 3/488

Ingrid Paus-Hasebrink / Klaus Neumann-Braun / Uwe Hasebrink / Stefan Aufenanger: Medienkindheit - Markenkindheit. Untersuchungen zur multimedialen Verwertung von Markenzeichen für Kinder. München: Kopaed, 2004 ............... 1/129

Hans-Jürgen Lüsebrink: Interkulturelle Kommunikation. Interaktion, Fremdwahrnehmung, Kulturtransfer. Stuttgart: Metzler, 2005 . . . . . . . . . 4/680

Jens Damm/Simona Thomas (Hrsg.): Chinese Cyberspaces. Technological Changes and Political Effects. London u. New York: Routledge, 2006 ... 3/489

Harald Gapski (Hrsg.): Medienkompetenzen messen? Verfahren und Reflexionen zur Erfassung von Schlüsselkompetenzen. Düsseldorf/München: kopaed, $2006 \ldots \ldots \ldots \ldots \ldots \ldots \ldots \ldots$. . . . . . . . . .

Rudolf Kammerl: Internetbasierte Kommunikation und Identitätskonstruktion. Selbstdarstellungen und Regelorientierungen 14- bis 16-jähriger Jugendlicher. Hamburg: Dr. Kovac, 2005 . . . . . . . . . . . 3/491

Klaus Arnold / Christoph Classen (Hrsg.): Zwischen Pop und Propaganda. Radio in der DDR. Berlin: Ch. Links, 2004

Michael Beuthner/Stephan Alexander Weichert (Hrsg.): Die Selbstbeobachtungsfalle. Grenzen und Grenzgänge des Medienjournalismus. Wiesbaden: VS Verlag für Sozialwissenschaften, 2005 . . . . . . 2/317

Roger Silverstone (Ed.): Media, Technology and Everyday Life in Europe. From Information to Communication. Aldershot: Ashgate, 2005 .... 2/318

Martin Wessner: Kontextuelle Kooperation in virtuellen Lernumgebungen. Lohmar: Eul, 2005 ..... 4/683 
Christina Ortner

Corinna Peil

Hans Peter Peters

Ulrich Riehm

Cornelia Rosebrock

Helge Rossen-Stadtfeld

Siegfried J. Schmidt

Rüdiger Schmitt-Beck

Irmela Schneider

Barbara Thomaß
Allan Brown / Robert G. Picard (Hrsg.): Digital Terrestrial Television in Europe. Mahwah, New Jersey/London: Lawrence Erlbaum Assoc. Publishers,

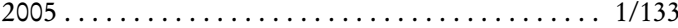

Joan Kristin Bleicher/Bernhard Pörksen (Hrsg.) Grenzgänger: Formen des New Journalism. Wiesbaden: VS, 2004 ... . . . . . . . . . . . . . . . . . . . . 4/684

Steffen Kolb: Mediale Thematisierung in Zyklen. Theoretischer Entwurf und empirische Anwendung. Köln: Herbert von Halem, 2005 . . . . . . . . . 3/493

Mirko Marr: Internetzugang und politische Informiertheit. Zur digitalen Spaltung der Gesellschaft. Konstanz: UVK, 2005 . . . . . . . . . . . . . . . . . . . 3/497

Bettina Hurrelmann/Susanne Becker/Irmgard Nickel-Bacon:Lesekindheiten. Familieund Lesesozialisation im historischen Wandel. Weinheim, München: Juventa, 2006 ........................ 4/688

Astrid Link: Unternehmensbeteiligungen öffentlich-rechtlicher Rundfunkanstalten. Öffentlichrechtlicher Programmauftrag und privatrechtliche Organisationsformen. Baden-Baden: Nomos Verlagsgesellschaft, $2005 \ldots \ldots \ldots \ldots \ldots \ldots \ldots . . . \ldots 3 / 500$

Bernhard Pörksen: Die Beobachtung des Beobachters. Eine Erkenntnistheorie der Journalistik. Konstanz: UVK, 2006

Werner Wirth / Edmund Lauf / Andreas Fahr (Hrsg.): Forschungslogik und -design in der Kommunikationswissenschaft. Band 1: Einführung, Problematisierungen und Aspekte der Methodenlogik aus kommunikationswissenschaftlicher Perspektive. Köln: Herbert von Halem, 2004 .............. . 1/134

Ulrike Schwab: Erzähltext und Spielfilm. Zur Ästhetik und Analyse der Filmadaption. Berlin: Lit, 2006 . . . . . . . . . . . . . . . . . . . . . . . . . . 4/691

Holger Schramm: Mood Management durch Musik. Die alltägliche Nutzung von Musik zur Regulierung von Stimmungen. Köln: Herbert von Halem,

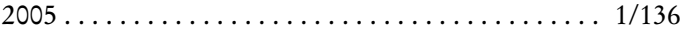

Christa-Maria Ridder / Wolfgang R. Langenbucher / Ulrich Saxer / Christian Steininger (Hrsg.): Bausteine einer Theorie des öffentlich-rechtlichen Rundfunks. Wiesbaden: VS Verlag für Sozialwissenschaften, 2005 . . . . . . . . . . . . . . . . . . . . . 2/320 
Jörg Ukrow

Andreas Wagenknecht

Stephan Alexander Weichert

Stephan Alexander Weichert

Carsten Winter

Reimar Zeh

Zeitschriftenlese

Literaturverzeichnis

English abstracts
Kristina Hopf: Jugendschutz im Fernsehen. Eine verfassungsrechtliche Prüfung der materiellen Jugendschutzbestimmungen. Frankfurt am Main,

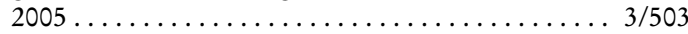

Friedrich Krotz: Neue Theorien entwickeln. Eine Einführung in die Grounded Theory, die Heuristische Sozialforschung und die Ethnographie anhand von Beispielen aus der Kommunikationsforschung. Köln: Herbert von Halem, 2005 . . . . . . . . . . 1/137

Kai Hafez: Mythos Globalisierung. Warum die Medien nicht grenzenlos sind. Wiesbaden: VS Verlag für Sozialwissenschaften, $2005 \ldots \ldots \ldots \ldots \ldots \ldots 2 / 322$

Günther Rager / Karola Graf-Szczuka / Gregor Hassemer / Stephanie Süper: Zeitungsjournalismus. Empirische Leserschaftsforschung. Konstanz,

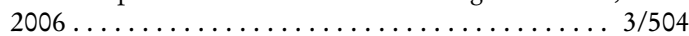

Andrea Grisold: Kulturindustrie Fernsehen. Zum Wechselverhältnis von Ökonomie und Massenmedien. Wien: Erhard Löcker Ges.m.b.H., 2004 . . . 2/324

Thomas Horky (Hrsg.): Erfahrungsberichte und Studien zur Fußball-Europameisterschaft. Hamburg: BoD GmbH, Norderstedt, 2005 ....... 2/327 $1 / 139,2 / 329,3 / 506,4 / 693$ $1 / 162,2 / 347,3 / 456,4 / 718$ $1 / 170,2 / 352,3 / 552,4 / 724$ 